\title{
Metabolic remodeling precedes mitochondrial outer membrane permeabilization in human glioma xenograft cells
}

\author{
SHIVANI PONNALA ${ }^{1}$, CHANDRAMU CHETTY $^{1}$, KRISHNA KUMAR VEERAVALLI ${ }^{1}$, \\ DZUNG H. DINH ${ }^{2}$, JEFFREY D. KLOPFENSTEIN ${ }^{2}$ and JASTI S. RAO ${ }^{1,2}$ \\ Departments of ${ }^{1}$ Cancer Biology and Pharmacology and ${ }^{2}$ Neurosurgery, \\ University of Illinois College of Medicine at Peoria, Peoria, IL 61605, USA
}

Received August 6, 2011; Accepted September 9, 2011

DOI: $10.3892 /$ ijo.2011.1255

\begin{abstract}
Glioma cancer cells adapt to changing microenvironment and shift from mitochondrial oxidative phosphorylation to aerobic glycolysis for their metabolic needs irrespective of oxygen availability. In the present study, we show that silencing MMP-9 in combination with uPAR/cathepsin B switch the glycolytic metabolism of glioma cells to oxidative phosphorylation (OXPHOS) and generate reactive oxygen species (ROS) to predispose glioma cells to mitochondrial outer membrane permeabilization. shRNA for MMP-9 and uPAR (pMU) as well as shRNA for MMP-9 and cathepsin B (pMC) activated complexes of mitochondria involved in OXPHOS and inhibited glycolytic hexokinase expression. The decreased interaction of hexokinase 2 with mitochondria in the treated cells indicated the inhibition of glycolysis activation. Overexpression of $\mathrm{Akt}$ reversed the pMU- and pMC-mediated OXPHOS to glycolysis switch. The OXPHOS un-coupler oligomycin A altered the expression levels of the Bcl-2 family of proteins; treatment with pMU or pMC reversed this effect and induced mitochondrial outer membrane permeabilization. In addition, our results show changes in mitochondrial pore transition to release cytochrome $\mathrm{c}$ due to changes in the VDAC-Bcl- $\mathrm{X}_{\mathrm{L}}$ and $\mathrm{BAX}-\mathrm{BAK}$ interaction with pMU and $\mathrm{pMC}$ treatments. Taken together, our results suggest that $\mathrm{pMU}$ and $\mathrm{pMC}$ treatments switch glioma cells from the glycolytic to the OXPHOS pathway through an inhibitory effect on Akt, ROS induction and an increase of cytosolic cytochrome $\mathrm{c}$ accumulation. These results demonstrate the potential of pMU and pMC as therapeutic candidates for the treatment of glioma.
\end{abstract}

Correspondence to: Dr Jasti S. Rao, Department of Cancer Biology and Pharmacology, University of Illinois College of Medicine at Peoria, One Illini Drive, Peoria, IL 61605, USA

E-mail: jsrao@uic.edu

Key words: aerobic glycolysis, MMP-9, uPAR, cathepsin B, oxidative phosphorylation, reactive oxygen species, Bcl-2 protein family, BAX, Bcl- $\mathrm{X}_{\mathrm{L}}$, cytochrome $\mathrm{c}$, apoptosis

\section{Introduction}

Glioblastoma multiforme (GBM) is a malignant tumor of the human brain. Primary treatment consists of surgical resection followed by radiotherapy and chemotherapy. Due to radioresistance and recurrence, patients with GBM typically have a median survival of 12-16 months. In well-established tumors, tumor cells exhibit chronic hypoxia with an increased rate of aerobic glycolysis and depleted functionality of mitochondrial oxidative phosphorylation (OXPHOS) (1-4). Hypoxia decreases the therapeutic potential of conventional radiotherapy and chemotherapy and results in poor outcome. Tumor cells modulate mitochondrial respiration under hypoxia to survive $(5,6)$. Previous attempts to oxygenate the tumor environment during therapy did not yield clinically convincing results $(7,8)$. This suggests that targeting the hypoxic tumor microenvironment along with tumor metabolism in glioma could improve the treatment strategies.

Akt signaling in metabolic remodeling has been studied extensively. The Akt signaling pathway modulates metabolic remodeling with ensuing tumor microenvironment modification and hypoxia inducing factor 1 alpha (HIF-1 $1 \alpha$ ) stabilization $(9,10)$. HIF-1 $\alpha$ regulates the expression of hexokinase (HK) 2, which helps in stabilization of aerobic glycolysis in GBM. Previous reports have shown that HK2 is overexpressed in $\operatorname{GBM}(11,12)$. Mitochondrial localization of HK2 is controlled by growth factor-induced oncogenic signaling from EGFR and PI3K/Akt activation, which are known to be overexpressed in GBM (13). HK2 binding to mitochondria through voltage-dependent anion channel (VDAC), thereby activates glycolysis and regulates the cytochrome c mediated intrinsic apoptosis $(14,15)$.

The mitochondrion plays a potential role in carcinogenesis because of its vital role in ATP generation for cellular processes, control of apoptosis, and generation of reactive oxygen species (ROS) through activation of metabolic pathways $(16,17)$. The glycolytic phenotype of solid tumors is associated with enhanced mitochondrial membrane polarization (i.e., hyperpolarized) and resistance to mitochondrial membrane permeability $(18,19)$. The $\mathrm{Bcl}-2$ family of proteins localizes to outer mitochondria and forms protein-permeable pores for the release of cytochrome $\mathrm{c}$ into the cytosol to initiate death signals, which mediate pro-apoptotic Bcl-2-associated X protein (BAX) translocation to the mitochondrial membrane (20). Pro-apoptotic BAX and 
anti-apoptotic $\mathrm{Bcl}-\mathrm{X}_{\mathrm{L}}$ respectively, facilitate and inhibit mitochondrial permeability transition pore opening and dissipate mitochondrial membrane potential (21).

Both OXPHOS dysfunction and aerobic glycolytic metabolism were shown to activate proteases such as cathepsins, metalloproteinases and urokinase plasminogen activator favored by micro-environmental acidosis, which promotes unimpeded proliferative and invasive cancer phenotype (22-24). Furthermore, previous studies have shown that mitochondrial mediated-ROS generation affects integrin signaling (25) and the expression and activation of matrix metalloproteinases $(26,27)$. In our earlier study, we have demonstrated that silencing of matrix metalloproteinase-9 (MMP-9) in combination with either urokinase plasminogen activator receptor (uPAR) or cathepsin B induced an anti-proliferative effect and inhibited pre-established tumor growth in vivo in nude mice $(28,29)$. In the present study, we show the effect of bicistronic MMP-9-uPAR (pMU) and MMP-9-cathepsin B (pMC) shRNA on metabolic reprogramming in glioma cells both in vitro and in vivo. Moreover, we demonstrate that our treatments induced metabolic remodeling and increased mitochondrial outer membrane permeabilization. The present study shows the potential use of our constructs to sensitize glioma cells by altering cell metabolism, increasing intracellular ROS levels, and initiating apoptotic cell death, and could improve overall patient outcome.

\section{Materials and methods}

Cell culture and transfection conditions. In the present study we used 4910 and 5310 human glioma xenograft cells that were kindly provided by Dr David James at University of California, San Francisco. These xenografts were highly invasive in mouse brains (30). Glioma cells were maintained in RPMI-1640 buffer supplemented with $10 \%$ fetal bovine serum, $50 \mu \mathrm{g} / \mathrm{ml}$ streptomycin and $50 \mathrm{U} / \mathrm{ml}$ penicillin in a humidified atmosphere containing $5 \% \mathrm{CO}_{2}$ at $37^{\circ} \mathrm{C}$. Glioma cells were transfected with shRNAs against MMP-9-uPAR (pMU), MMP-9-cathepsin B (pMC), scrambled vector (pSV), dominant-negative Akt (dnAkt) or constitutively activated Akt (myr-Akt). Plasmids containing the above constructs were transfected using FuGENE ${ }^{\circledR}$ HD reagent (Roche Diagnostics, Indianapolis, IN) according to the manufacturer's instructions. pMU, pMC and pSV were used as described previously (29). Both dnAkt and myr-Akt were obtained from Addgene (Carlsbad, CA). After transfection, cells were incubated in serum containing medium for a minimum of $60 \mathrm{~h}$.

We used antibodies for the oxidative phosphorylation cocktail (MitoSciences, Eugene, OR), Akt, pAkt, HK1, HK2, VDAC1, Bcl-X $\mathrm{L}_{\mathrm{L}}, \mathrm{BAX}, \mathrm{BAK}$, cytochrome c, IgG and GAPDH (all from Santa Cruz Biotechnology, Santa Cruz, CA). $\alpha$-tubulin and COX-IV were obtained from Cell Signaling (Danvers, MA).

Preparation of mitochondrial and cytoplasmic extracts. Cells were treated with pSV, pMU, pMC, myr-Akt or dnAkt for $72 \mathrm{~h}$, collected and washed twice with ice-cold PBS. The mitochondrial and cytoplasmic extracts were prepared using the Thermo Fisher Scientific Mitochondria Isolation Kit (Hanover Park, IL) according to the manufacturer's instructions and used in further analyses.
Western blotting. Western blotting was performed by lysing the cells with radio-immunoprecipitation assay buffer (1\% IGEPAL, $20 \mathrm{mM}$ Tris, $150 \mathrm{mM} \mathrm{NaCl}, 5 \mathrm{mM}$ EDTA, $0.1 \mathrm{mg} / \mathrm{ml}$ aprotinin, and $1 \mathrm{mM}$ phenylmethyl sulfonyl fluoride). Then, equal amounts of protein fraction were resolved over SDS-PAGE with a $4.5 \%$ stacking gel. Samples were electrophoresed and electroblotted onto a nitrocellulose membrane. Western blot analysis was performed with a 1:1000 dilution of primary antibody. Western blot analysis of the relative levels of the five OXPHOS complexes in mitochondrial preparations against Complex I subunit NDUFB8, Complex II subunit $30 \mathrm{kDa}$, Complex III subunit Core 2, Complex IV subunit II, and ATP synthase subunit $\alpha$ were performed with MitoProfile ${ }^{\circledR}$ Total OXPHOS Human WB Antibody Cocktail according to the manufacturer's instructions (MitoSciences, Eugene, OR). Immunoreactive bands were visualized after processing the blots with horseradish peroxidase (HRP)-conjugated secondary antibody. Signals were detected using the ECL Western blotting detection system (Pierce, Rockford, IL)

Immunofluorescence imaging of glioma cells. We used MitoTracker Red CMXRos (Molecular Probes, Carlsbad, CA) reagent to stain mitochondria of both transfected and control cells. The cells were incubated with $300 \mathrm{nM}$ of stain for $40 \mathrm{~min}$. After washing twice with PBS for 20 min each, cells were fixed in $10 \%$ buffered formalin for $10 \mathrm{~min}$. After washing with PBS two more times, the fixed cells were permeabilized with $0.3 \%$ Triton-X100 for $10 \mathrm{~min}$. Permeabilized cells were washed twice with PBS and blocked with goat serum for $30 \mathrm{~min}$ and then incubated with 1:100 ratio of anti-HK2 or anti-Bcl-X $\mathrm{L}_{\mathrm{L}}$ antibody in goat serum for $1 \mathrm{~h}$. After washing the cells twice with PBS, cells were incubated with 1:200 dilution of Alexa Fluor 488 goat anti-mouse or goat anti-rabbit immunoglobulin (molecular probes) in goat serum for $1 \mathrm{~h}$. Nuclei were stained with DAPI, which was mixed with immune-mount. Fluorescent images were taken with a confocal fluorescent microscope (Olympus BX61 Fluoview, Minneapolis, MN).

Determination of intracellular ROS. ROS levels were analyzed using Total ROS/Superoxide Detection kit from Enzo Life Sciences, Inc. (Plymouth Meeting, PA). The non-fluorescent, cell-permeable total ROS detection dye was added to both transfected and control cells followed by incubation for $45 \mathrm{~min}$. The dye reacted directly with a wide range of reactive species, such as hydrogen peroxide, peroxynitrite and hydroxyl radicals, yielding a green fluorescent product indicative of cellular production of different ROS/RNS types. The cells were washed twice with PBS in a volume sufficient to cover the cell monolayer and analyzed using a fluorescent microscope and/or flow cytometry equipped with standard green filter $(490 / 525 \mathrm{~nm}$ or $488 \mathrm{~nm}$ laser). Appropriate positive control samples induced with Pyocyanin exhibit bright green fluorescence in the cytoplasm. Cells pre-treated with the ROS inhibitor do not demonstrate any green fluorescence signal upon induction.

Cell viability assay. To determine the effect of oligomycin A on cell viability, we carried out the trypan blue exclusion assay. Cells were treated with oligomycin $48 \mathrm{~h}$ after transfection for $24 \mathrm{~h}$. Then, the cells were suspended in an equal volume of trypan blue stain $(0.4 \% \mathrm{w} / \mathrm{v})$ and incubated for $5 \mathrm{~min}$. Finally, 
cells were counted using the Countess Automated Cell Counter (Invitrogen, Carlsbad, CA).

Immunohistochemistry. Paraffin-embedded brain sections (5 $\mu \mathrm{m}$ thick) from control and treatment groups were deparaffinized following standard protocols. Antigen retrieval was performed by treating the sections with citrate buffer at $95^{\circ} \mathrm{C}$ for 15-20 min followed by hydrogen peroxide treatment for $30 \mathrm{~min}$. The sections were rinsed with PBS and blocked with $1 \%$ BSA in PBS to prevent non-specific staining and further incubated overnight with primary antibodies (1:100 dilution) at $4^{\circ} \mathrm{C}$. The sections were then incubated with either HRP- or Alexa Fluor 488-conjugated secondary antibodies for $1 \mathrm{~h}$ at room temperature followed by incubation with either DAB/DAPI for $15 \mathrm{~min}$. The sections treated with HRP-conjugated secondary antibody were counterstained with hematoxylin to visualize the nucleus, mounted and observed under a light microscope. The sections treated with Alexa Fluor 488 secondary antibody were mounted and observed under a confocal microscope (Olympus BX61 Fluoview).

Densitometry. ImageJ software (National Institutes of Health) was used to quantify band intensity. Data represent intensities relative to the indicated loading control.

Statistical analysis. All data are presented as mean \pm standard deviation (SD) of at least three independent experiments. Statistical comparisons were performed using Graph Pad Prism software (version 3.02). Bonferroni's post hoc test (multiple comparison tests) was used to compare any statistical significance between groups. Differences in the values were considered significant at $\mathrm{p}<0.05$.

\section{Results}

pMU and pMC modulate mitochondrial membrane transition by activating $O X P H O S$. In our earlier studies, we have demonstrated the efficiency of our constructs and the role of pMU and pMC in downregulating the DNA damage repair mechanism in glioma cells both in vitro and in vivo. Both pMU and pMC induced cell death in glioma cells with accumulated DNA damage. Based on this observation, we hypothesized that pMU and pMC might contribute to DNA damage accumulation through mitochondrial ROS generation mediated by metabolic remodeling. Malignant glioma predominantly shifts to aerobic glycolysis, which shuts down OXPHOS even under a high oxygen level, thus preventing ROS generation. To investigate the switch from the glycolytic to oxidative phosphorylation mode of glioma metabolism, we analyzed the expression of relative levels of the five OXPHOS complexes in mitochondrial preparations against Complex I subunit NDUFB8, Complex II subunit $30 \mathrm{kDa}$, Complex III subunit Core 2, Complex IV subunit II, and ATP synthase subunit $\alpha$. There was a significant increase in the expression of the five OXPHOS complexes with pMU and pMC treatments as compared to control and pSV (Fig. 1A). COX-IV indicated purity of mitochondrial fractions (Fig. 1A). These results indicated stimulation of the OXPHOS pathway in treated glioma cells. To determine whether mitochondrial OXPHOS pathway activation was associated with ROS generation, we examined intracellular ROS levels using the Invitrogen ROS detection kit. As shown in Fig. 1B and C, the levels of intracellular ROS in pMU- and pMC-transfected cells were significantly higher when compared with control and pSV-transfected glioma cells.

Silencing MMP-9 in combination with $u P A R /$ cathepsin B regulates key glycolytic enzymes. A classical metabolic adaptation of cancer cells to aerobic glycolysis in contrast to oxidative phosphorylation for its energy requirements is largely observed. This is supported by the frequent high levels of glycolysis-associated proteins, a key stimulus is hexokinase (HK) expression in cancer cells. We determined the effect of pMU and pMC treatments on HK1 and HK2 expression by Western blotting. pMU and pMC treatments increased HK1 expression as compared to control and pSV-transfected cells (Fig. 2A). Further, HK2 expression decreased markedly in pMU-and pMC-transfected glioma cells as compared to control and pSV (Fig. 2A). Interaction of HK with voltage-dependent anion-selective channel protein 1 (VDAC1) on mitochondria is associated with activation of glycolysis. In view of this, we next determined the expression levels of VDAC1 in glioma cells using Western blotting. No change in expression of VDAC1 was observed in pMU- and $\mathrm{pMC}$-treated cells as compared to control and $\mathrm{pSV}$. The degree of mitochondria-bound HK2 was assessed by immunofluorescence using mitochondria organelle specific MitoTracker red stain. Decreased mitochondria-bound HK2 was observed (white spots) in pMU- and pMC-transfected cells as compared to control and pSV-treated glioma cells (Fig. 2B). These findings suggested that when treated with pMU and pMC, glioma cells switch from the glycolytic pathway to the OXPHOS pathway and generate high intracellular ROS.

pMU and pMC mediates glycolysis to OXPHOS switch via Akt signaling. Several studies have confirmed a link between Akt pathway activation and increased glycolysis activation and HIF-1 $\alpha$ expression $(31,32)$. In our earlier study, we observed significant reduction in the expression and stabilization of HIF- $1 \alpha$ in glioma cells after pMU and pMC treatments. Therefore, we determined the effect of pMU and pMC transcriptional suppression on Akt. As shown in Fig. 3A, pMU and pMC transfection markedly inhibited the expression and phosphorylation of Akt (Ser-473). To determine the contribution of the Akt pathway and its downstream effector on OXPHOS activation, 5310 and 4910 glioma cells were transiently transfected with either constitutively active-Akt (myr-Akt) or dominant-negative Akt (dnAkt) before pMU, pMC or pSV transfection. As shown in Fig. 3B, ectopic expression of constitutive active-Akt restored Akt phosphorylation and expression of OXPHOS-related complexes. However, this was reversed by dnAkt in pMU- and pMC-transfected cells (Fig. 3B). These results confirm Akt-mediated activation of OXPHOS pathway in pMU- and pMC-treated glioma cells.

pMU and pMC regulate mitochondrial outer membrane permeabilization by altering the Bcl-2 family of proteins. We next sought to determine the sequential signaling events connecting mitochondrial membrane permeabilization-OXPHOS complex overexpression and mitochondrial membrane permeabilizationapoptosis initiation. Inhibition of OXPHOS by metabolic remodeling of tumor cells to aerobic glycolysis induces resistance to apoptosis by suppressing the activation of the pro-apoptotic 


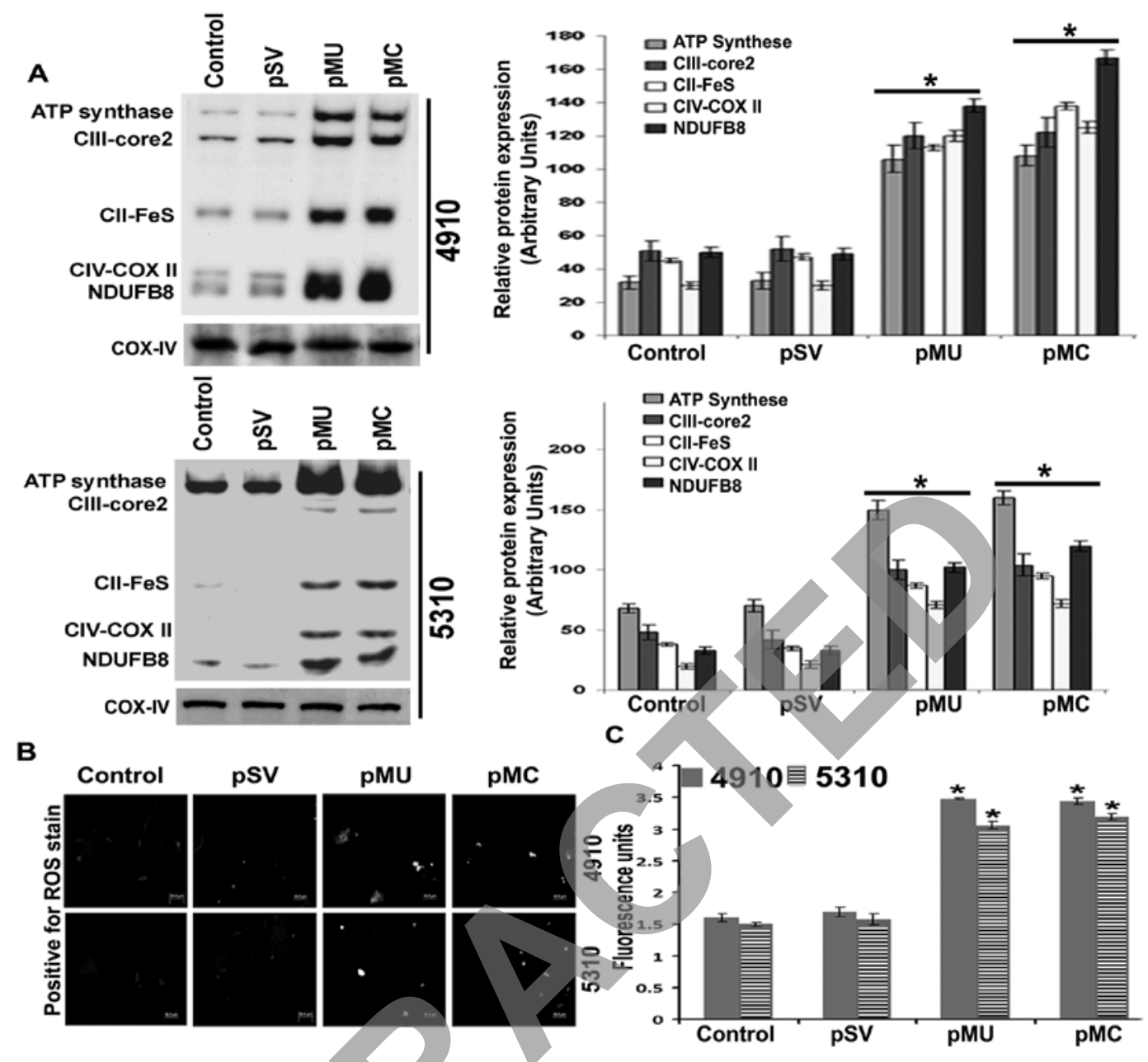

Figure 1.pMU and pMC stimulate OXPHOS and ROS production in human glioma xenograft cells. (A) Mitochondrial fractions were assessed for OXPHOS-associated protein complex levels by Western blotting. COX-IV showed purity of mitochondrial fractions. Densitometry analysis show relative protein expression. (B) After $72 \mathrm{~h}$ of control, pSV, pMU and pMC treatments the cells were washed with PBS and treated with ROS detection reagent for 45 min. The degree of ROS generated was observed in live cells after adding ROS detection reagent using fluorescence microscope. (C) Glioma cells were plated in 96-well plates, transfected with control, pSV, pMU and $\mathrm{pMC}$ for $72 \mathrm{~h}$, and assessed for the changes in ROS levels using ROS detection reagent. The fluorometric reading was obtained using Flex station and a representative graph is shown. Results are representative of three independent experiments (columns: mean of three experiments; bars, $\pm \mathrm{SD}$ ). "p $<0.05$, difference between control/ $\mathrm{pSV}$ and $\mathrm{pMU} / \mathrm{pMC}$.

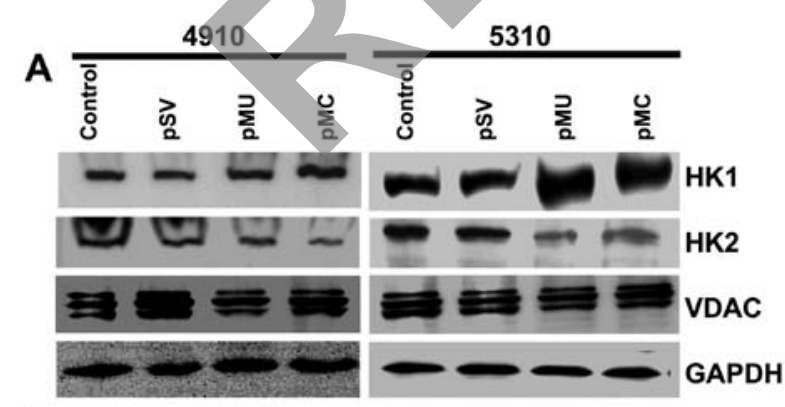

B

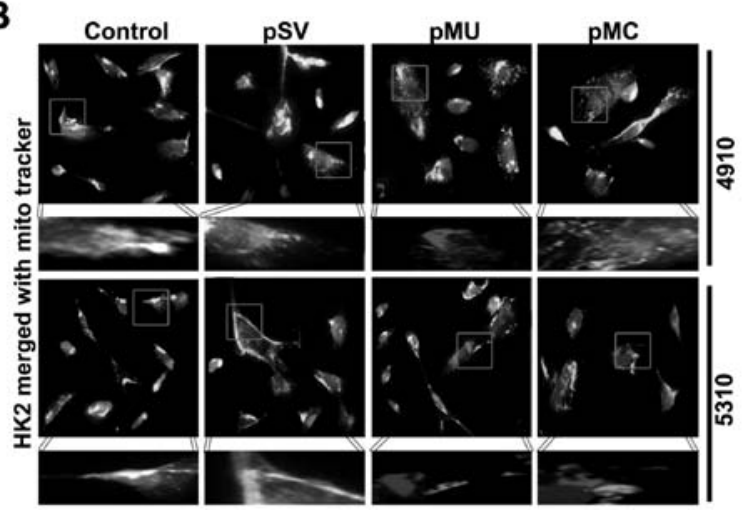

Bcl-2 family members, BAX and BAK (33). Therefore, we examined the effects of $\mathrm{pMU}$ and $\mathrm{pMC}$ transfection on the expression of the Bcl-2 family of proteins. pMU- and pMC-transfected glioma cells inhibited anti-apoptotic $\mathrm{Bcl}-\mathrm{X}_{\mathrm{L}}$ expression and increased pro-apoptotic BAX expression (Fig. 4A). To further validate the effect of mitochondrial metabolic remodeling on the expression of the Bcl-2 family of proteins, we incubated cells with oligomycin A (5-10 $\mu \mathrm{M})$, a known ATP synthase inhibitor for $12 \mathrm{~h}$. The inhibition of ATP synthase by oligomycin A restored Bcl- $\mathrm{X}_{\mathrm{L}}$ expression and depleted BAX expression in glioma cells (Fig. 4B). Further, transfection of glioma cells with pMU and $\mathrm{pMC}$ for $48 \mathrm{~h}$ followed by incubation with oligomycin $\mathrm{A}$ reversed $\mathrm{pMU} / \mathrm{pMC}$ induced pro-apoptotic effects; decreased

Figure 2. pMU and pMC treatments inhibit glycolysis in glioma. (A) Cell lysates were assessed for HK1, HK2 and VDAC levels by Western blotting. GAPDH served as a loading control. (B) Immunofluorescence analysis show HK2 binding to mitochondria (MitoTracker red stain). Interaction is shown as bright white spots. Results are representative of three independent experiments. 
A
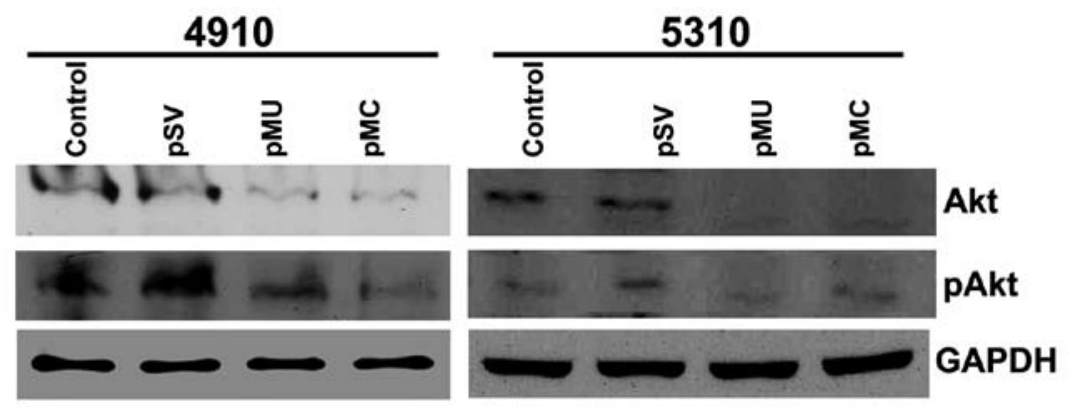

B

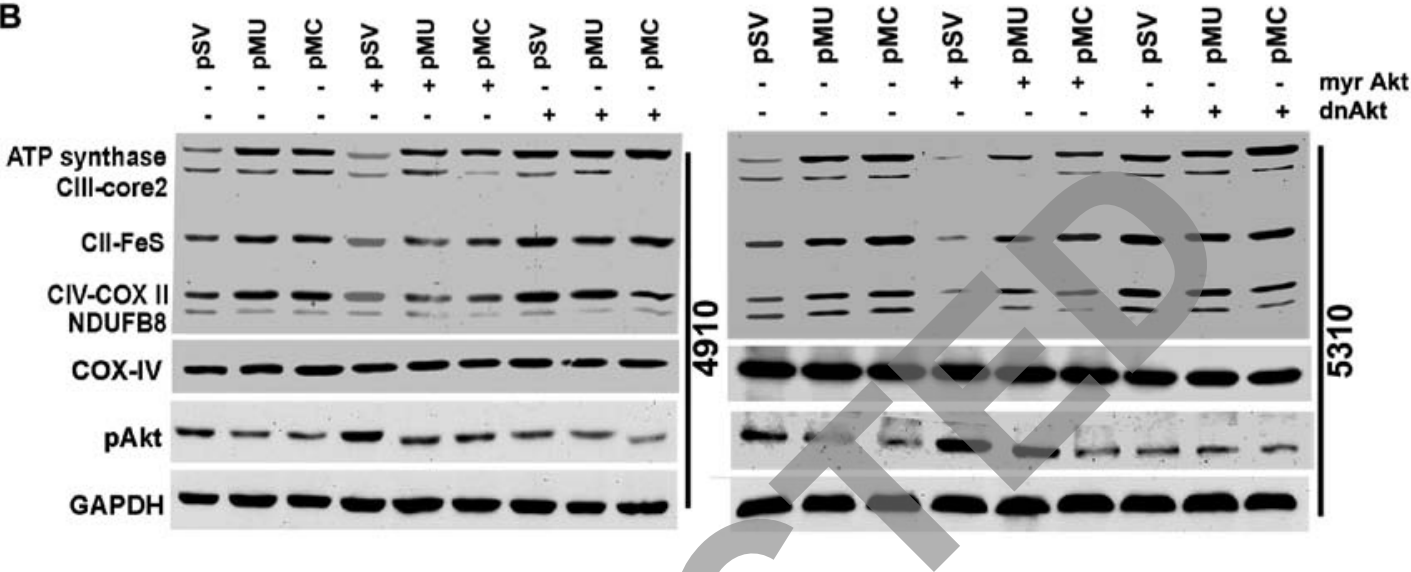

Figure 3. pMU and pMC mediate Akt-dependent OXPHOS activation in glioma. (A) Cell lysates were assessed for pAkt and Akt levels by Western blotting. (B) Glioma cells were transfected with $\mathrm{pSV}$, pMU or pMC for $48 \mathrm{~h}$ followed by transfection with myr-Akt or dnAkt and cultured for another $24 \mathrm{~h}$. Cells were harvested and lysed, and fractions of mitochondrial and total cell lysates were collected separately. These fractions were used for Western blot analysis of OXPHOS complexes and pAkt. COX-IV indicated purity of mitochondrial fractions. GAPDH served as a loading control for total cell lysates. Results are representative of three independent experiments.

Bcl- $\mathrm{X}_{\mathrm{L}}$ expression and increased BAX expression (Fig. 4D). Cell viability assay showed $\mathrm{pMU}$ and $\mathrm{pMC}$ significantly reduced the cell population in oligomycin-treated glioma cells (Fig. 4C).

Mitochondrial cytochrome c release is regulated by $p M U$ and pMC in glioma xenograftcells. The aerobic glycolytic phenotype has been shown to enhance mitochondrial membrane polarization (i.e., hyperpolarization) and to oppose mitochondrial membrane permeability $(18,19)$. Further, OXPHOS metabolism is required for the activation of pro-apoptotic Bcl-2 families, $\mathrm{BAX}$ and BAK expression (33). The interactions between BAK, $\mathrm{BAX}, \mathrm{Bcl}-\mathrm{X}_{\mathrm{L}}$ and VDAC are known to regulate mitochondrial membrane permeabilization. So, we examined the effects of pMU and pMC transfection on the interaction of these proteins by immunocytochemistry and immunoprecipitation analyses. pMU- and pMC-treated glioma cells showed reduced interaction of $\mathrm{Bcl}-\mathrm{X}_{\mathrm{L}}$ with mitochondria as compared to untreated cells (Fig. 5A). Immunoprecipitation studies showed reduced association between VDAC and Bcl- $\mathrm{X}_{\mathrm{L}}$ and increased association of BAX with BAK (Fig. 5B). Our results show the possible role of increased $\mathrm{BAX}$ and $\mathrm{BAK}$ interaction to form transition pores that allow cytochrome $\mathrm{c}$ release from mitochondria, which precedes activation of caspases that initiate the apoptotic cascade. Transfection of glioma cells with pMU- and pMCshowed increased levels of cytosolic cytochrome c (Fig. 5C). Taken together, these results suggest that the pre-mitochondrial membrane permeabilization effect of metabolic remodeling follows post-mitochondrial membrane permeabilization by mitochondrial transition pore opening, cytochrome c release and activation of death signaling.
In vivo expression of pAkt, OXPHOS complexes, $B c l-X_{L}, B A X$ and cytochrome $c$. In our previous study, we evaluated the effect of pMU and pMC on tumor formation in vivo after intracranial implantation of glioma cells in nude mice. The tumors that arose were treated with intratumoral injection of either pMU or pMC. Tumor growth was monitored in mice using an in vivo imaging system (Xenogen, IVIS, Hopkinton, MA). There was a significant decrease in the tumor volume of mice treated with either pMU or pMC when compared with untreated control mice $(28,29)$. Reduction of the tumor volume observed was $>85 \%(28,29)$.

To determine whether either pMU or pMC caused Aktmediated glycolysis to OXPHOS shift predisposed glioma cells to apoptosis as mediated by the Bcl-2 family of proteins in vivo, tumor sections were immunoassayed for pAkt, BAX, Bcl- $\mathrm{X}_{\mathrm{L}}$ OXPHOS complex of protein cocktail, and cytochrome c. Fig. 6A and B show results consistent with our in vitro results; tumor sections from pMU- and $\mathrm{pMC}$-treated mice had decreased staining for $\mathrm{pAkt}$ and $\mathrm{Bcl}-\mathrm{X}_{\mathrm{L}}$ and increased cytochrome c, BAX and OXPHOS complex expression.

\section{Discussion}

In GBM patients upregulated aerobic glycolysis was shown using stereotactic microdialysis, wherein three times more lactate:pyruvate ratio was measured in the tumor area as compared to normal brain $(34,35)$. Several studies show that glycolytic acidosis of tumor microenvironment elevates extracellular matrix, urokinase plasminogen expression, tumor proliferation and invasive property; however, no studies 
A
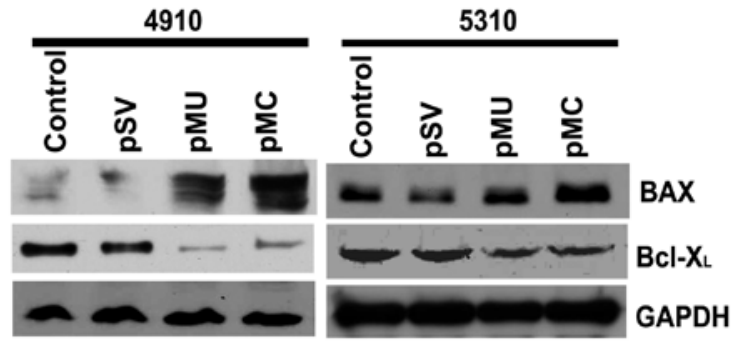

B

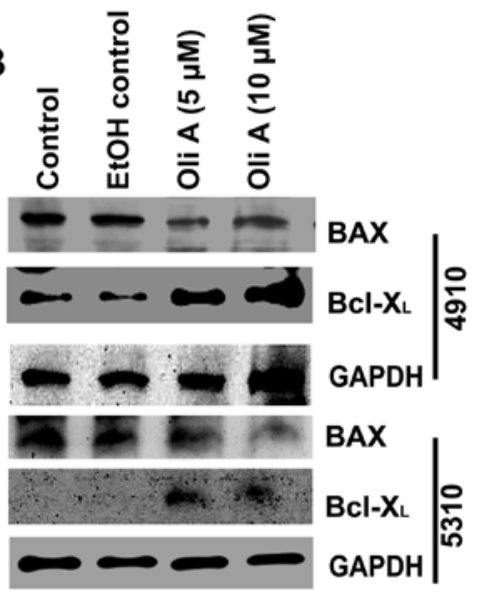

$\mathbf{C}_{120}$

$120 \cdot \square 4910 \square 53310$

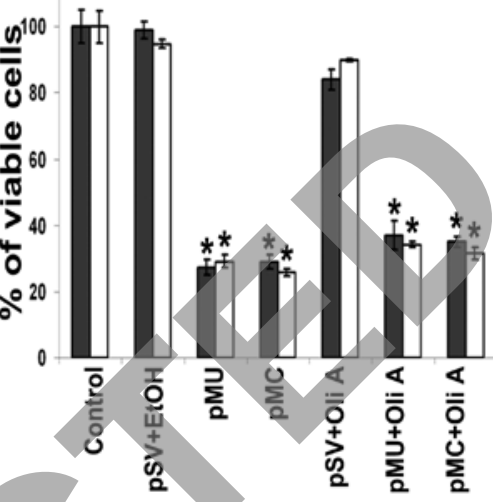

D
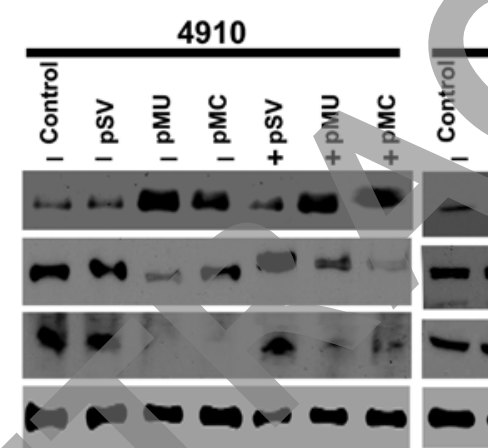

5310
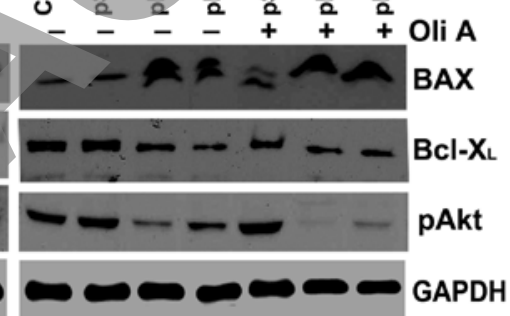

Figure 4. pMU and pMC regulate pro- and anti-apoptotic Bcl-2-related proteins. (A) Cell lysates were assessed for BAX and Bcl- $\mathrm{X}_{\mathrm{L}}$ by Western blotting. GAPDH served as a loading control. (B) Cells were treated with OXPHOS uncoupler, oligomycin A for $12 \mathrm{~h}$. The cell lysates were prepared for Western blot analysis of BAX and Bcl- $\mathrm{X}_{\mathrm{L}}$ levels. GAPDH served as a loading control. (C) Graph shows the percent of viable cells as assessed by trypan blue cell viability assay. " $\mathrm{p}<0.05$, difference between control $/ \mathrm{pSV}$ and $\mathrm{pMU} / \mathrm{pMC}$. (D) Glioma cells were transfected with $\mathrm{pSV}$, pMU or pMC for $48 \mathrm{~h}$ and the cells were then treated with oligomycin A for $12 \mathrm{~h}$. To assess BAX, Bcl- $\mathrm{X}_{\mathrm{L}}$ and pAkt levels, total cell lysates were prepared. GAPDH served as a loading control. Results are representative of three independent experiments.

have shown the functional mechanism by which extracellular matrix plays a role in metabolic remodeling in tumor cells. In the present study, we show that MMP-9-uPAR (pMU) and MMP-9-cathepsin B (pMC) bicistronic shRNAs induced the switch from aerobic glycolytic metabolism to oxidative phosphorylation and generated ROS in glioma tumor cells. Also, we show the signaling mechanisms that direct pMU- and pMC-induced metabolic reprogramming and ROS-mediated mitochondrial cytochrome $\mathrm{c}$ release into cytosol in glioblastoma tumor cells.

MMP-9 in combination with uPAR or cathepsin B induced the overexpression of OXPHOS protein complexes in vitro as shown by immunoblot analysis. Furthermore, ROS generation proved switch to OXPHOS pathway in pMU- and pMC-transfected glioblastoma cells. We also show that metabolic activation of the mitochondrial OXPHOS pathway of pMU- and pMCtransfected glioblastoma cells depends on Akt regulation. Our immunoblot results shows pMU- and pMC-transfected glioma cells had decreased expression of Akt. Extracellular signaling and oncogenic activation of growth factor/PI3K/Akt stimulates glycolysis by glucose uptake and stabilizes HIF-1 $\alpha$ activation $(9,10,36,37)$. Our earlier study shows that $\mathrm{pMU} / \mathrm{pMC}$ inhibits HIF-1 $\alpha$ expression and activity in glioma cells. We further tested the effect of pMU and pMC silencing on Akt-mediated regulation of OXPHOS protein expression. Constitutive activation of Akt (myr-Akt) inhibited the pMU- and pMC-induced OXPHOS protein complexes. Conversely, dominant negative expression of Akt elevated pMU- and pMC-mediated mitochondrial oxidation. Our study clearly shows that Akt mediated mitochondrial OXPHOS regulation in MMP-9-, uPAR- and cathepsin B-silenced cells.

We next investigated how MMP-9 in combination with uPAR or cathepsin B silencing modulates glycolysis inhibition in glioma cells. Cancer cells take up high glucose, which 


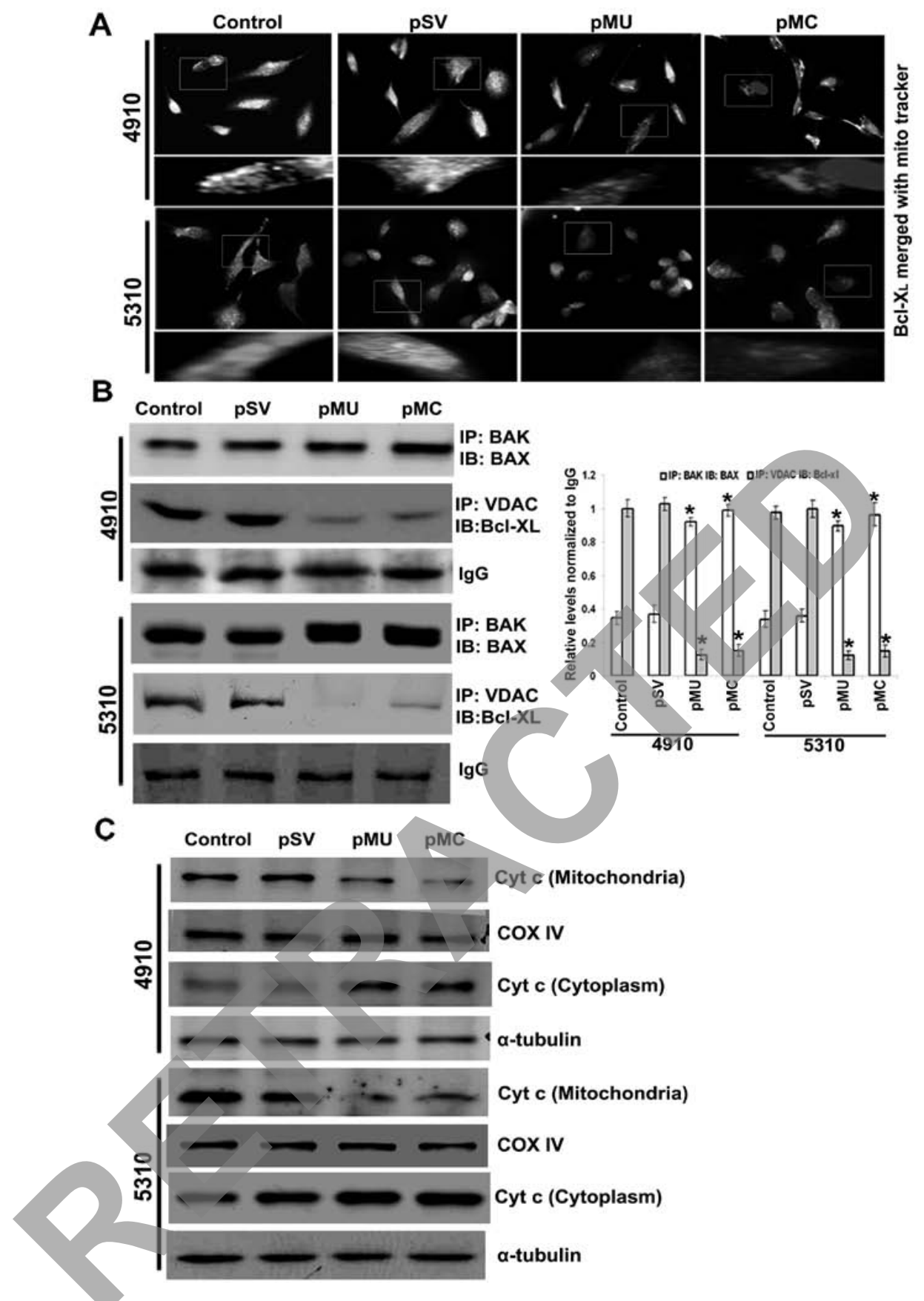

Figure 5. pMU and pMC induce mitochondrial cytochrome c release. (A) Immunofluorescence analysis shows Bcl- $\mathrm{X}_{\mathrm{L}}$ binding to mitochondria (MitoTracker red stain). Interaction is shown as bright white spots. (B) Cell lysates were assessed for interaction between VDAC1 and Bcl- $\mathrm{X}_{\mathrm{L}}$ and BAX and BAK using immunoprecipitation. Densitometric analysis show interaction of BAX-BAK and VDAC- Bcl- $\mathrm{X}_{\mathrm{L}}$. IgG showed equal loading. Results are representative of three independent experiments (columns, mean of three experiments; bars, \pm SD). " $\mathrm{p}<0.05$, difference between control $/ \mathrm{pSV}$ and pMU/pMC. (C) Cells were harvested and lysed, and fractions of mitochondrial and cytosolic were collected separately. These fractions were assessed by Western blot analysis using anti-cytochrome $\mathrm{c}$ antibody. COX-IV and $\alpha$-tubulin showed purity of mitochondrial and cytosolic fractions, respectively.

is phosphorylated to glucose-6-phosphate by key glycolytic enzymes, hexokinase 1 (HK1) and 2 (HK2). Compared to HK1, HK2 plays an important role in establishing the aerobic glycolytic phenotype in GBM $(12,38)$. HK2 expression is regulated by HIF- $1 \alpha$ activation and plays an important role in glucose conversion in hypoxia. Stable silencing of HK2 switches GBM tumor cell metabolism from aerobic glycolysis to mitochondrial respiration by increasing OXPHOS protein expression and also shows anti-tumorigenicity in subcutaneous and intracranial xenograft models (39). Evidence also suggests that Akt pathway activation can translocate glycolytic enzyme HK2 to the outer mitochondrial membrane through its interaction with the voltage dependent anion channel (VDAC) and regulate the intrinsic apoptotic pathway $(14,40)$. Our study shows that pMU- and pMC-silenced cells decreased HK2 expression. Alternatively, HK1 is overexpressed in pMU- and pMC-treated cells compared to its low levels in GBM (41-43). Furthermore, we show that mitochondrial-bound HK2 decreased as assessed by immunoblotting and immunocytochemical analyses. These findings suggest that pMU and pMC regulate glycolytic enzymes 

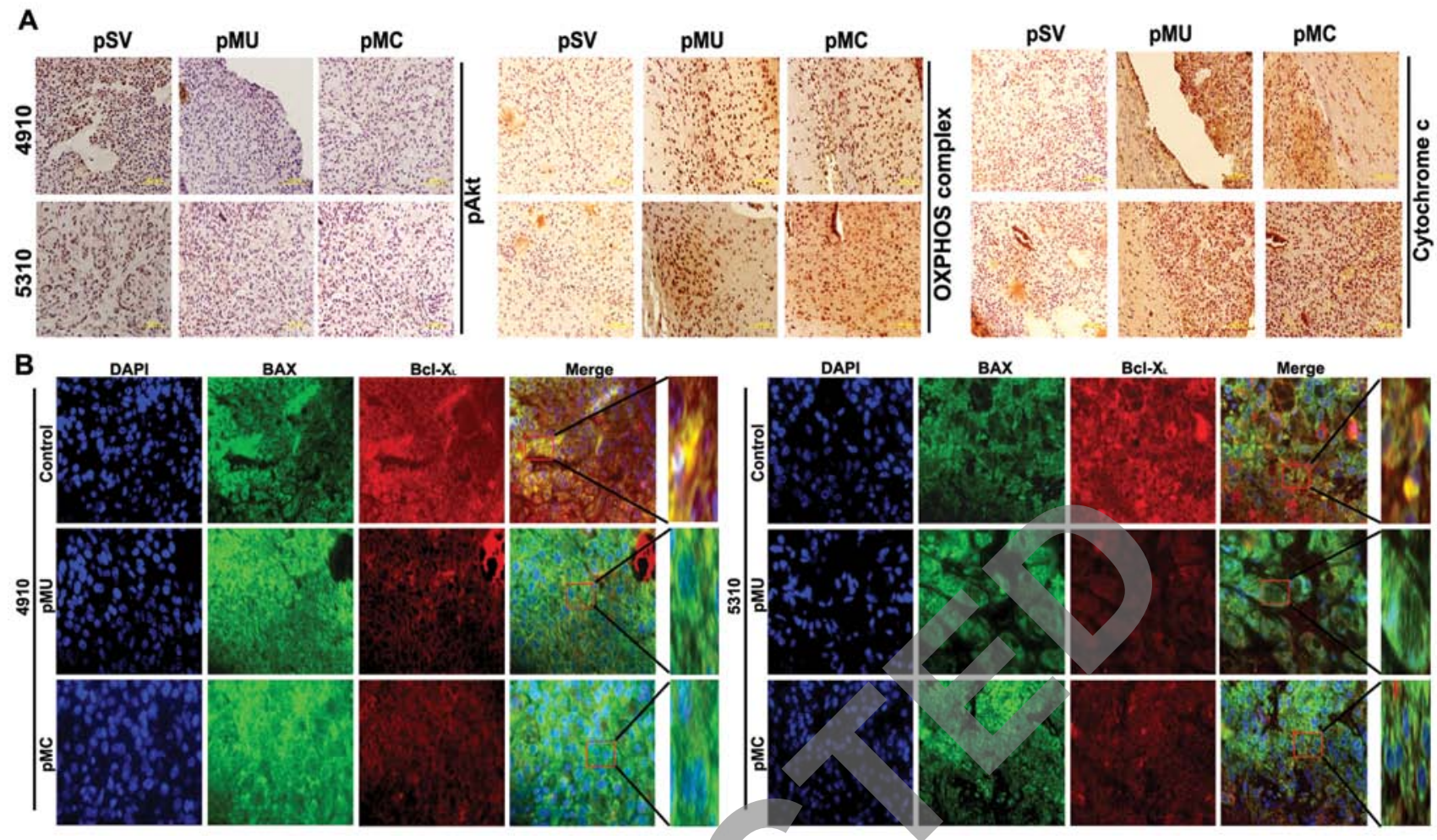

Figure 6. Silencing MMP-9 in combination with uPAR/cathepsin B alters expression of pAkt, OXPHOS complex, cytochrome c, BAX and Bcl- $\mathrm{X}_{\mathrm{L}}$ in pre-established intracranial tumors. A total of six animals were studied in each group. (A) Immunohistochemical analysis of pAkt, OXPHOS complex and cytochrome c expression. (B) Expression of Bcl- $\mathrm{X}_{\mathrm{L}}$ and BAX in brain sections as described in Materials and methods. BAX (green) and Bcl- $\mathrm{X}_{\mathrm{L}}$ (red) are double-labeled for the same section.

leading to the inhibition of mitochondrial-bound HK2 signaling in glycolysis and anti-apoptosis.

Our results also reveal that the pMU- and pMC-induced metabolic switch from aerobic glycolysis to OXPHOS precedes elevated cytoplasmic cytochrome c levels. Mitochondrial oxidative respiration byproduct ROS is not only implicated in damage of cellular components but also plays an important role in mitochondrial membrane permeabilization, which initiates intracellular apoptotic or pro-survival signaling (44-46). Metabolic remodeling to glycolysis in tumor cells decreases ROS and lactate, thereby protecting tumor cells from oxidative stress and inhibiting cellular death (47-50). Mitochondrial membrane potential transition to release cytochrome $\mathrm{c}$, a prerequisite to apoptosis initiation, depends on the ratio of the expression levels of pro- and anti-apoptotic Bcl-2 family of proteins (51). Our study shows that pMU- and pMC-silenced glioma cells had decreased anti-apoptotic Bcl- $\mathrm{X}_{\mathrm{L}}$ and increased pro-apoptotic BAX expression. Studies have shown that the OXPHOS pathway stimulated BAX and BAK expression and triggered apoptotic cell death by disparate death stimuli (33). In addition, specific inhibition of ATP-synthase in human carcinoma cells suppressed tumor necrosis factor-induced apoptosis (52). It has been shown that BAX-induced cell death in yeast depends on ATP synthase and is reversed by oligomycin treatment (53). Other mechanisms of anti-apoptotic action of oligomycin include blockage of dimerization of BAX in calphostin C-induced apoptosis, inhibition of cytochrome c in some BAX-independent apoptosis, and inhibition of apoptosis induced by anti-cancer drugs etoposide and dexamethasone (53-57). Our study shows that ATP synthase inhibition by oligomycin A increased anti-apoptotic Bcl- $\mathrm{X}_{\mathrm{L}}$ expression and decreased pro-apoptotic BAX expression in glioma. Moreover, cell viability assay showed pMU and pMC retarded the oligomycin A effect and decreased glioma cell viability. Furthermore, we showed that pMU and pMC treatments in glioma reversed the effect of oligomycin $\mathrm{A}$ and induced Akt inhibition. These findings suggest that pMU and pMC modulate the OXPHOS pathway in ROS generation and trigger mitochondrial membrane permeabilization by $\mathrm{Bcl}-2$ expression.

The Bcl-2 family of proteins have been shown to interact with VDAC to facilitate their anti-apoptotic effect by regulating the mitochondrial permeability transition pore (MPTP) opening to release cytochrome c (58). Overexpresssed $\mathrm{Bcl}-2$ has been associated with resistance to anti-cancer drugs $(59,60)$. One of the major stimuli for mitochondria membrane permeabilization and efflux of cytochrome c release is BAX and BAK oligomerization (61-63). In our study immunoprecipitation analysis showed reduced interaction of VDAC with $\mathrm{Bcl}-\mathrm{X}_{\mathrm{L}}$ and increased association of BAK and BAX in shRNA-transfected glioma cells. We observed a decrease in $\mathrm{Bcl}-\mathrm{X}_{\mathrm{L}}$ localization with mitochondria in pMU- and pMC-transfected glioma cells. Our data indicate that increased BAX and BAK release pro-apoptotic mitochondrial cytochrome $\mathrm{c}$ into cytosol in pMU- and pMCsilenced glioma cells. Once in the cytosol, cytochrome c protein activates caspase-dependent and -independent programmed cell death $(19,28)$.

Our in vivo study showed that silencing MMP-9 in combination with either UPAR or cathepsin B resulted in decreased immunoreactivity of pAkt and increased immunoreactivity of OXPHOS complexes and cytosolic cytochrome c. Further, co-localization study of BAX and $\mathrm{Bcl}-\mathrm{X}_{\mathrm{L}}$ showed that the 
increased $\mathrm{BAX}$ and $\mathrm{Bcl}-\mathrm{X}_{\mathrm{L}}$ interaction in tumor sections from mice that received $\mathrm{pMU}$ or $\mathrm{pMC}$ treatments compared to tumor sections from mice that received pSV treatments. Our findings add to the current therapeutic agents targeting cancer cell death by generating excess ROS (64) and also have advantages over current agents targeting HK2 (e.g., 3-bromopyruvate, 2-deoxyglucose) which show limited clinical potential in GBM due to non-specificity.

In conclusion, we show that increased intracellular ROS levels generated by OXPHOS activation by pMU/pMC signaling via the Akt pathway participate as a crucial factor for the initiation of apoptosis in glioma cells. ROS may lead to depletion of anti-oxidants in mitochondria and expose mitochondria to oxidative stress, further contributing to the rapid decrease in mitochondrial membrane potential by altering the expression levels of the Bcl-2 family proteins and the release of cytochrome c into the cytosol, which is followed by caspase activation $(28,65)$. Thus, these results indicate a possible mitochondria-ROS mediated pMU/pMC-induced apoptosis of human glioma xenograft cells.

\section{Acknowledgments}

This study was supported by a grant from the National Institute of Neurological Disorder and Stroke (N.I.N.D.S.) (NS047699 to J.S.R.). The contents of this manuscript are solely the responsibility of the authors and do not necessarily represent the official views of National Institute of Health (N.I.H.). The authors thank Shellee Abraham for manuscript preparation, and Diana Meister and Sushma Jasti for manuscript review.

\section{References}

1. Baggetto LG: Deviant energetic metabolism of glycolytic cancer cells. Biochimie 74: 959-974, 1992.

2. Osthus RC, Shim H, Kim S, Li Q, Reddy R, Mukherjee M, Xu Y, Wonsey D, Lee LA and Dang CV: Deregulation of glucose transporter 1 and glycolytic gene expression by c-Myc. J Biol Chem 275: 21797-21800, 2000.

3. Pedersen PL: Tumor mitochondria and the bioenergetics of cancer cells. Prog Exp Tumor Res 22: 190-274, 1978

4. Shim H, Chun YS, Lewis BC and Dang CV: A unique glucosedependent apoptotic pathway induced by c-Myc. Proc Natl Acad Sci USA 95: 1511-1516, 1998.

5. lalunis-Turner MJ, Franko AJ and Parliament MB: Modulation of oxygen consumption rate and vascular endothelial growth factor mRNA expression in human malignant glioma cells by hypoxia. Br J Cancer 80: 104-109, 1999.

6. Franko AJ, Parliament MB, lalunis-Turner MJ and Wolokoff BG: Variable presence of hypoxia in M006 human glioma spheroids and in spheroids and xenografts of clonally derived sublines. Br J Cancer 78: 1261-1268, 1998.

7. Kaanders JH and van der Kogel AJ: Erythropoietin to treat anaemia in patients with head and neck cancer. Lancet 363: 78-79, 2004.

8. Vaupel P: Tumor microenvironmental physiology and its implications for radiation oncology. Semin Radiat Oncol 14: 198-206, 2004.

9. Denko NC: Hypoxia, HIF1 and glucose metabolism in the solid tumour. Nat Rev Cancer 8: 705-713, 2008.

10. Manning BD and Cantley LC: AKT/PKB signaling: navigating downstream. Cell 129: 1261-1274, 2007.

11. Dong S, Nutt CL, Betensky RA, Stemmer-Rachamimov AO, Denko NC, Ligon KL, Rowitch DH and Louis DN: Histologybased expression profiling yields novel prognostic markers in human glioblastoma. J Neuropathol Exp Neurol 64: 948-955, 2005.

12. Wolf A, Agnihotri S and Guha A: Targeting metabolic remodeling in glioblastoma multiforme. Oncotarget 1: 552-562, 2010.
13. Mellinghoff IK, Wang MY, Vivanco I, Haas-Kogan DA, Zhu S, Dia EQ, Lu KV, Yoshimoto K, Huang JH, Chute DJ, Riggs BL, Horvath S, Liau LM, Cavenee WK, Rao PN, Beroukhim R, Peck TC, Lee JC, Sellers WR, Stokoe D, Prados M, Cloughesy TF, Sawyers CL and Mischel PS: Molecular determinants of the response of glioblastomas to EGFR kinase inhibitors. N Engl J Med 353: 2012-2024, 2005.

14. Majewski N, Nogueira V, Bhaskar P, Coy PE, Skeen JE, Gottlob K, Chandel NS, Thompson CB, Robey RB and Hay N: Hexokinasemitochondria interaction mediated by Akt is required to inhibit apoptosis in the presence or absence of Bax and Bak. Mol Cell 16: 819-830, 2004.

15. Pastorino JG, Shulga N and Hoek JB: Mitochondrial binding of hexokinase II inhibits Bax-induced cytochrome c release and apoptosis. J Biol Chem 277: 7610-7618, 2002.

16. Dellagiacoma G, Sbarbati A, Rossi M, Zancanaro C, Benati D, Merigo F, Baldassarri A and Boicelli A: Brown adipose tissue: magnetic resonance imaging and ultrastructural studies after transplantation in syngeneic rats. Transplant Proc 24: 2986, 1992.

17. Singh KK: Mitochondrial DNA mutations. In: Aging, Disease and Cancer. Springer, New York, NY, 1998.

18. Chen LB: Mitochondrial membrane potential in living cells. Annu Rev Cell Biol 4: 155-181, 1988.

19. Kroemer G, Galluzzi L and Brenner C: Mitochondrial membrane permeabilization in cell death. Physiol Rev 87: 99-163, 2007.

20. Goping IS, Gross A, Lavoie JN, Nguyen M, Jemmerson R, Roth K, Korsmeyer SJ and Shore GC: Regulated targeting of BAX to mitochondria. J Cell Biol 143: 207-215, 1998.

21. Susin SA, Zamzami N and Kroemer G: Mitochondria as regulators of apoptosis: doubt no more. Biochim Biophys Acta 1366: 151-165, 1998.

22. Gatenby RA and Gillies RJ: Why do cancers have high aerobic glycolysis? Nat Rev Cancer 4: 891-899, 2004.

3. Swietach P, Vaughan-Jones RD and Harris AL: Regulation of tumor $\mathrm{pH}$ and the role of carbonic anhydrase 9. Cancer Metastasis Rev 26: 299-310, 2007.

24. Van Waveren C, Sun Y, Cheung HS and Moraes CT: Oxidative phosphorylation dysfunction modulates expression of extracellular matrix-remodeling genes and invasion. Carcinogenesis 27: 409-418, 2006.

25. Svineng G, Ravuri C, Rikardsen O, Huseby NE and Winberg JO: The role of reactive oxygen species in integrin and matrix metalloproteinase expression and function. Connect. Tissue Res 49: 197-202, 2008.

26. Kumar B, Koul S, Khandrika L, Meacham RB and Koul HK: Oxidative stress is inherent in prostate cancer cells and is required for aggressive phenotype. Cancer Res 68: 1777-1785, 2008.

27. Nelson KK and Melendez JA: Mitochondrial redox control of matrix metalloproteinases. Free Radic Biol Med 37: 768-784, 2004.

28. Chetty C, Lakka SS, Bhoopathi P, Gondi CS, Veeravalli KK, Fassett D, Klopfenstein JD, Dinh DH, Gujrati M and Rao JS: Urokinase plasminogen activator receptor and/or matrix metalloproteinase-9 inhibition induces apoptosis signaling through lipid rafts in glioblastoma xenograft cells. Mol Cancer Ther 9: 2605-2617, 2010.

29. Veeravalli KK, Chetty C, Ponnala S, Gondi CS, Lakka SS, Fassett D, Klopfenstein JD, Dinh DH, Gujrati M and Rao JS: MMP-9, uPAR and cathepsin B silencing downregulate integrins in human glioma xenograft cells in vitro and in vivo in nude mice. PLoS One 5: e11583, 2010.

30. Giannini C, Sarkaria JN, Saito A, Uhm JH, Galanis E, Carlson BL, Schroeder MA and James CD: Patient tumor EGFR and PDGFRA gene amplifications retained in an invasive intracranial xenograft model of glioblastoma multiforme. Neurooncology 7: 164-176, 2005.

31. Gordan JD and Simon MC: Hypoxia-inducible factors: central regulators of the tumor phenotype. Curr Opin Genet Dev 17: 71-77, 2007.

32. Semenza GL: Targeting HIF-1 for cancer therapy. Nat Rev Cancer 3: 721-732, 2003.

33. Tomiyama A, Serizawa S, Tachibana K, Sakurada K, Samejima H, Kuchino Y and Kitanaka C: Critical role for mitochondrial oxidative phosphorylation in the activation of tumor suppressors Bax and Bak. J Natl Cancer Inst 98: 1462-1473, 2006.

34. Tabatabaei P, Bergstrom P, Henriksson R and Bergenheim AT: Glucose metabolites, glutamate and glycerol in malignant glioma tumours during radiotherapy. J Neurooncol 90: 35-39, 2008 . 
35. Simon DK and O'Leary DD: Relationship of retinotopic ordering of axons in the optic pathway to the formation of visual maps in central targets. J Comp Neurol 307: 393-404, 1991.

36. Buzzai M, Bauer DE, Jones RG, Deberardinis RJ, Hatzivassiliou G, Elstrom RL and Thompson CB: The glucose dependence of Akt-transformed cells can be reversed by pharmacologic activation of fatty acid beta-oxidation. Oncogene 24 : 4165-4173, 2005.

37. Elstrom RL, Bauer DE, Buzzai M, Karnauskas R, Harris MH, Plas DR, Zhuang H, Cinalli RM, Alavi A, Rudin CM and Thompson CB: Akt stimulates aerobic glycolysis in cancer cells. Cancer Res 64: 3892-3899, 2004.

38. Pedersen PL, Mathupala S, Rempel A, Geschwind JF and Ko YH: Mitochondrial bound type II hexokinase: a key player in the growth and survival of many cancers and an ideal prospect for therapeutic intervention. Biochim Biophys Acta 1555: 14-20, 2002.

39. Wolf A, Agnihotri S, Munoz D and Guha A: Developmental profile and regulation of the glycolytic enzyme hexokinase 2 in normal brain and glioblastoma multiforme. Neurobiol Dis 44: 84-91, 2011.

40. Gottlob K, Majewski N, Kennedy S, Kandel E, Robey RB and Hay N: Inhibition of early apoptotic events by Akt/PKB is dependent on the first committed step of glycolysis and mitochondrial hexokinase. Genes Dev 15: 1406-1418, 2001.

41. Lowry OH, Berger SJ, Carter JG, Chi MM, Manchester JK, Knor $\mathbf{J}$ and Pusateri ME: Diversity of metabolic patterns in human brain tumors: enzymes of energy metabolism and related metabolites and cofactors. J Neurochem 41: 994-1010, 1983.

42. Marzatico F, Curti D, Dagani F, Silvani V, Gaetani P, Butti G and Knerich R: Enzymes related to energy metabolism in human gliomas. J Neurosurg Sci 30: 129-132, 1986.

43. Oudard S, Poirson F, Miccoli L, Bourgeois Y, Vassault A, Poisson M, Magdelenat H, Dutrillaux B and Poupon MF: Mitochondria-bound hexokinase as target for therapy of malignant gliomas. Int J Cancer 62: 216-222, 1995 .

44. Azad MB, Chen Y and Gibson SB: Regulation of autophagy by reactive oxygen species (ROS): implications for cancer progression and treatment. Antioxid Redox Signal 11: 777-790, 2009.

45. Simon HU, Haj-Yehia A and Levi-Schaffer F: Role of reactive oxygen species (ROS) in apoptosis induction. Apoptosis 5: 415-418, 2000.

46. Wochna A, Niemczyk E, Kurono C, Masaoka M, Kedzior J, Slominska E, Lipinski M and Wakabayashi T: A possible role of oxidative stress in the switch mechanism of the cell death mode from apoptosis to necrosis-studies on rho0 cells. Mitochondrion 7: 119-124, 2007.

47. Ahmad A, Ahmad S, Schneider BK, Allen CB, Chang LY and White CW: Elevated expression of hexokinase II protects human lung epithelial-like A549 cells against oxidative injury. Am J Physiol Lung Cell Mol Physiol 283: L573-L584, 2002.

48. Brand KA and Hermfisse U: Aerobic glycolysis by proliferating cells: a protective strategy against reactive oxygen species. FASEB J 11: 388-395, 1997.

49. Kim JW, Tchernyshyov I, Semenza GL and Dang CV: HIF-1mediated expression of pyruvate dehydrogenase kinase: a metabolic switch required for cellular adaptation to hypoxia. Cell Metab 3: 177-185, 2006.
50. Zhang H, Gao P, Fukuda R, Kumar G, Krishnamachary B, Zeller KI, Dang CV and Semenza GL: HIF-1 inhibits mitochondrial biogenesis and cellular respiration in VHL-deficient renal cell carcinoma by repression of C-MYC activity. Cancer Cell 11: 407-420, 2007.

51. Green DR and Reed JC: Mitochondria and apoptosis. Science 281: 1309-1312, 1998.

52. Shchepina LA, Pletjushkina OY, Avetisyan AV, Bakeeva LE, Fetisova EK, Izyumov DS, Saprunova VB, Vyssokikh MY, Chernyak BV and Skulachev VP: Oligomycin, inhibitor of the F0 part of $\mathrm{H}^{+}$-ATP-synthase, suppresses the TNF-induced apoptosis. Oncogene 21: 8149-8157, 2002.

53. Matsuyama S, Xu Q, Velours J and Reed JC: The mitochondrial F0F1-ATPase proton pump is required for function of the proapoptotic protein Bax in yeast and mammalian cells. Mol Cell 1: 327-336, 1998 .

54. Eguchi Y, Shimizu S and Tsujimoto Y: Intracellular ATP levels determine cell death fate by apoptosis or necrosis. Cancer Res 57: $1835-1840,1997$.

55. Goldstein JC, Waterhouse NJ, Juin P, Evan GI and Green DR: The coordinate release of cytochrome $\mathrm{c}$ during apoptosis is rapid, complete and kinetically invariant. Nat Cell Biol 2: 156-162, 2000.

56. Ikemoto H, Tani E, Ozaki I, Kitagawa $\mathrm{H}$ and Arita N: Calphostin $\mathrm{C}$-mediated translocation and integration of Bax into mitochondria induces cytochrome c release before mitochondrial dysfunction. Cell Death Differ 7: 511-520, 2000.

57. Leist M, Single B, Castoldi AF, Kuhnle S and Nicotera P: Intracellular adenosine triphosphate (ATP) concentration: a switch in the decision between apoptosis and necrosis. J Exp Med 185: 1481-1486, 1997.

58. Azoulay-Zohar H, Israelson A, bu-Hamad S and ShoshanBarmatzV:In self-defence: hexokinase promotes voltage-dependent anion channel closure and prevents mitochondria-mediated apoptotic cell death. Biochem J 377: 347-355, 2004.

59. Adams JM and Cory S: The Bcl-2 apoptotic switch in cancer development and therapy. Oncogene 26: 1324-1337, 2007.

60. Alexander HK, Booy EP, Xiao W, Ezzati P, Baust H and Los M: Selected technologies to control genes and their products for experimental and clinical purposes. Arch Immunol Ther Exp (Warsz.) 55: 139-149, 2007.

61. Antignani A and Youle RJ: How do Bax and Bak lead to permeabilization of the outer mitochondrial membrane? Curr Opin Cell Biol 18: 685-689, 2006.

62. Wei MC, Zong WX, Cheng EH, Lindsten T, Panoutsakopoulou V, Ross AJ, Roth KA, MacGregor GR, Thompson CB and Korsmeyer SJ: Proapoptotic BAX and BAK: a requisite gateway to mitochondrial dysfunction and death. Science 292: 727-730, 2001.

63. Wei MC, Lindsten T, Mootha VK, Weiler S, Gross A, Ashiya M, Thompson CB and Korsmeyer SJ: tBID, a membrane-targeted death ligand, oligomerizes BAK to release cytochrome c. Genes Dev 14: 2060-2071, 2000

64. Agostinelli E and Seiler N: Non-irradiation-derived reactive oxygen species (ROS) and cancer: therapeutic implications. Amino Acids 31: 341-355, 2006.

65. Hamanaka RB and Chandel NS: Mitochondrial reactive oxygen species regulate cellular signaling and dictate biological outcomes. Trends Biochem Sci 35: 505-513, 2010. 